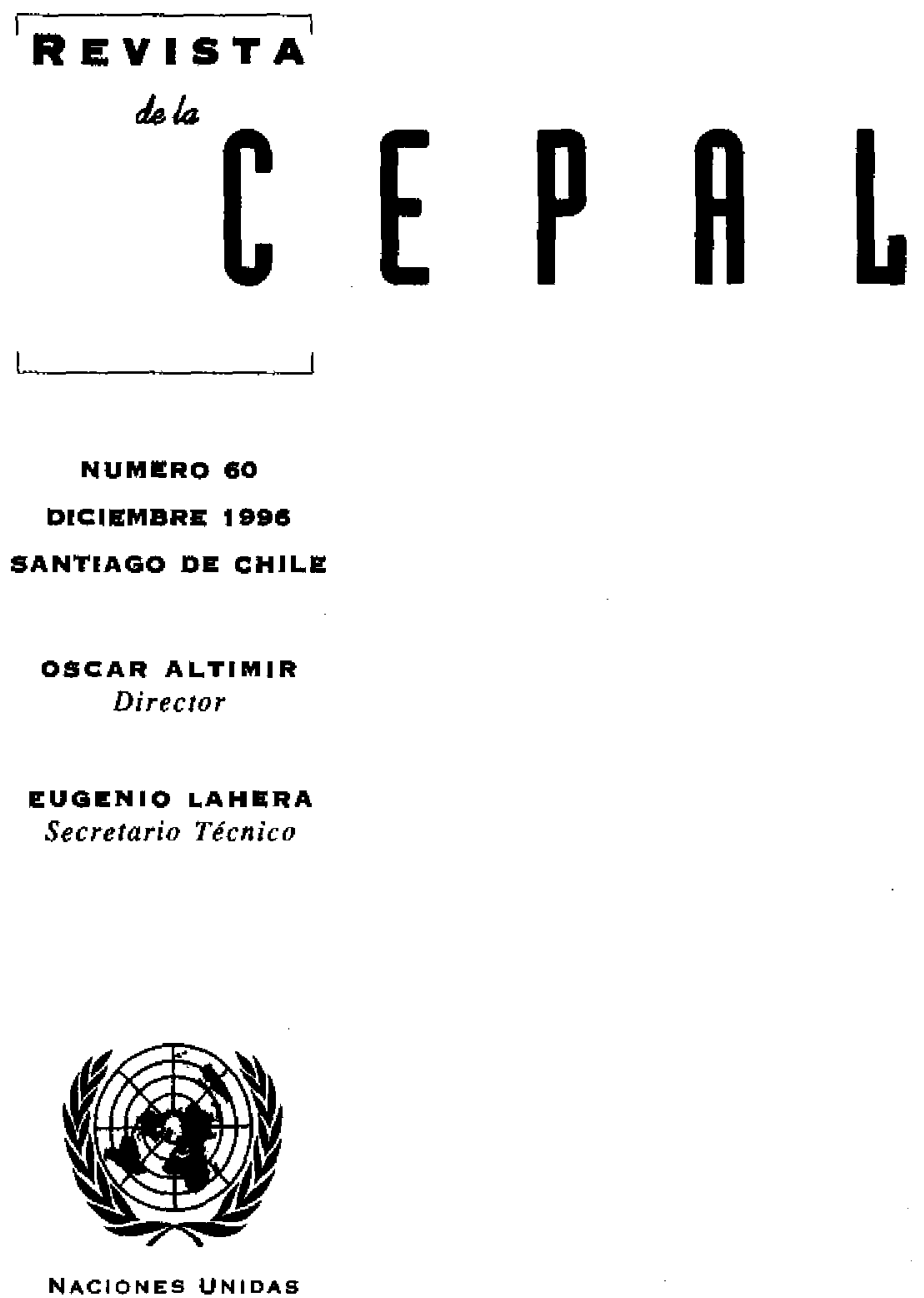


La evolución de las ideas y las políticas para el desarrollo

Gert Rosenthal

Políticas macroeconómicas para el crecimiento

Ricardo Ffrench-Davis

Flujos de capitales: lecciones a partir de la experiencia chilena

Eduardo Aninat, Christian Larraín

La transformación del desarrollo industrial de América Latina

José Miguel Benavente, Gustavo Crespi, Jorge Katz, Giovanni Stumpo

Las reformas de las pensiones en América Latina y la posición de los organismos internacionales

Carmelo Mesa-Lago

Aportes de la antropología aplicada al desarrollo campesino

John Durston

Prospección de la biodiversidad: potencialidades para los países en desarrollo

Julie M. Feinsilver

La inversión extranjera y el desarrollo competitivo en América Latina y el Caribe

Alejandro C. Vera-Vassallo

La crisis del peso mexicano

Stephany Griffith-Jones 


\section{Flujos de capitales: lecciones a partir de la experiencia chilena}

\section{Eduardo Aninat}

Ministro de Hacienda de Chile

\section{Christian Larraín}

Coordinador de

Finanzas Internacionales,

Ministerio de

Hacienda de Chile
El presente artículo examina el esquema de regulación de capitales utilizado en la economía chilena durante los últimos años. Para ello describe, en primer lugar, los factores determinantes de los movimientos de capitales internacionales en el período reciente, así como el papel del sistema financiero en la intermediación de dichos flujos. Luego considera la política chilena de regulación de flujos de capitales, que respondió al dilema de cómo conciliar las reducciones de la inflación con la mantención de un tipo de cambio real compatible con la competitividad de las exportaciones. Sus instrumentos de política incluyen la intervención del Banco Central, que se refleja en un fuerte aumento de las reservas internacionales, aparejada con operaciones de esterilización a través del mercado abierto. Dados los límites de esta estrategia, el proceso ha ido acompañado por graduales devaluaciones reales del peso. Asimismo, desde mediados de 1991 Chile ha aplicado controles no cuantitativos de capital, buscando desincentivar las entradas de recursos de corto plazo. Otro rasgo distintivo de la política chilena de regulación de capitales ha sido el de otorgar mayor flexibilidad a las salidas de capital. El artículo concluye que esta política ha favorecido la consecución de altas tasas de ahorro interno y contribuido a disminuir la vulnerabilidad de la economía chilena. Otros factores importantes de estos logros han sido una política fiscal austera y la existencia de adecuadas exigencias de carácter prudencial en el sistema bancario. 


\section{I}

\section{Antecedentes}

Transcurrido más de un año de la devaluación del peso mexicano y del llamado "efecto tequila" que afectó en distinto grado a las economías de la región, todavía pesan en algunas de ellas los efectos recesivos e inflacionarios de esa coyuntura, ligados a una brusca reversión de los flujos de capitales internacionales.

Hay consenso en que esos movimientos generan efectos favorables, como los de complementar el ahorro interno -que suele ser bajo en economías emergentes-, diversificar riesgos y minimizar los costos de ajustes derivados de discrepancias entre el ingreso y el gasto.

No obstante, la experiencia muestra que sin una política que privilegie la mantención de los equilibrios macroeconomicos fundamentales ( fundamentals) ${ }^{1}$ mediante una regulación adecuada, la entrada de fuertes corrientes financieras internacionales puede tener efectos nocivos para el desempeño de la economía receptora. En particular, de la experiencia del bienio 19941995, que afectó con singular fuerza a algunos países de la región, se desprende que la reversión de esas corrientes también puede acarrear costos significativos en términos de actividad, desempleo e inflación.

En la propia historia económica chilena existen antecedentes de un manejo de capitales poco riguroso, en un contexto de liberalización financiera, durante el período 1979-1982. Esto tuvo consecuencias claramente perniciosas a través de una fuerte apreciación cambiaria, que distorsionó la asignación de recursos entre bienes transables y no transables y la disponibi- lidad de fondos para el financiamiento de la inversion, entre otros aspectos.

Vemos así que la libre circulación de los recursos financieros internacionales tiene efectos positivos y negativos. El desafío que enfrentan las políticas públicas es el de potenciar los primeros sin comprometer los equilibrios macroeconómicos, manteniendo las variables fundamentales en sus valores tendenciales y dentro de un esquema regulatorio que garantice la estabilidad del sistema financiero en el contexto de un rol de intermediación acrecentado.

Dicho desafío se agudizo a comienzos de los años noventa, al producirse una fuerte afluencia de capitales hacia economías emergentes, a la cual no escapó la chilena. En el caso de Chile, la aplicación de una política activa de regulación de los movimientos de capitales internacionales ha permitido mantener un exitoso desempeño económico (cuadro 1). Incluso en 1995, año en que el crecimiento global de América Latina se redujo a aproximadamente $0 \%$, la economía chilena exhibió resultados favorables, alcanzando por primera vez en más de tres décadas un crecimiento del PIB superior a la inflación: $8,5 \%$ contra $8,2 \%$. Las perspectivas para 1996 son de crecimiento e inflación en magnitudes de $6.5 \%$.

En este artículo se describe el esquema de regulación de los flujos de capitales internacionales utilizado en la economía chilena durante los años noventa, y de esa experiencia se extraen algunas lecciones en materia de política.

\section{II}

\section{Factores determinantes de los movimientos de capitales internacionales en los años noventa}

\section{Aspectos conceptuales}

La afluencia de capitales internacionales desempeña un papel decisivo en el proceso de desarrollo de las eco-

$\square$ Los autores agradecen los comentarios de Ricardo Ffrench-Davis y Heinz Rudolph. nomías. Entre las funciones que tradicionalmente se le atribuye destaca la de movilizar capital hacia países en desarrollo, lo que permite a éstos complemen-

\footnotetext{
${ }^{1}$ Concepto que envuelve tanto la solidez y estabilidad de estos equilibrios como la correspondencia de los precios relativos claves en su valor tendencial a mediano plazo.
} 
CUADRO 1

Chile: Resultados macroeconómicos

(Porcentajes)

\begin{tabular}{lcccc}
\hline & $\begin{array}{c}\text { Crecimiento } \\
\text { del PIB real }\end{array}$ & Inflación & Desempleo & $\begin{array}{c}\text { Inversión } \\
\text { como \% del PIB }\end{array}$ \\
\hline 1987 & 6.6 & 21.5 & 9.3 & 22.2 \\
1988 & 7.3 & 12.7 & 8.1 & 22.8 \\
1989 & 9.9 & 21.4 & 6.3 & 25.5 \\
1990 & 3.3 & 27.3 & 6.0 & 26.3 \\
1991 & 7.3 & 18.7 & 6.5 & 24.5 \\
1992 & 11.0 & 12.7 & 4.9 & 26.8 \\
1993 & 6.3 & 12.2 & 4.6 & 28.8 \\
1994 & 4.2 & 8.9 & 5.9 & 26.8 \\
$1995^{\mathrm{a}}$ & 8.5 & 8.2 & 5.4 & 27.6 \\
$1996^{\mathrm{b}}$ & 6.5 & 6.5 & $\ldots$ & $\ldots$ \\
\hline
\end{tabular}

Fuente: Banco Central de Chile.

a Cifras preliminares.

${ }^{b}$ Estimaciones.

tar el ahorro interno, elevar sus niveles de inversion y estimular su crecimiento. Asimismo, la movilidad de los capitales favorece la diversificación de los riesgos, y permite minimizar los costos de ajustes intertemporales derivados de discrepancias entre el ingreso y el gasto.

Sin embargo, ciertas imperfecciones que caracterizan el funcionamiento de los mercados de capital pueden dar origen a externalidades y desembocar en disparidades entre las valoraciones privadas y las sociales. Como se sabe, el teorema fundamental de la economía de bienestar - que todo equilibrio competitivo conduce a un óptimo paretiano- está basado en dos supuestos claves: primero, que la información debe ser exógena, es decir, no debe verse afectada por las acciones de agente alguno en el mercado, y segundo, que en éste debe existir una oferta de una gama completa de productos. Estos supuestos claramente no se cumplen en el caso de los mercados financieros, puesto que proveer información es una de sus funciones esenciales. Por otra parte, algunos riesgos pueden permanecer al margen de la cobertura de los seguros y no existen grandes incentivos para que las instituciones bancarias proporcionen financiamiento de largo plazo (Stiglitz,1993).

Es característico de los mercados financieros que los prestadores e inversionistas consideren básicamente la rentabilidad que esperan obtener, subvalorando los excedentes totales que genera la empresa cuyo financiamiento se considera. Por lo tanto, los proyectos con la más alta rentabilidad esperada para el prestador pueden no coincidir con los de más alto rendimiento, a pesar de lo cual recibirán financiación. Asimismo, si la información es imperfecta los mercados financieros pueden no equilibrarse. En estos mercados, los dispuestos a pagar más pueden no ser aquellos que signifiquen un mayor retorno esperado para el prestador. De hecho, dicha rentabilidad puede caer si las tasas de interés suben, ya que la probabilidad de insolvencia tiende a elevarse. Como resultado, puede haber racionamiento de crédito: aun cuando exista un exceso de demanda crediticia, los prestadores pueden evitar el alza de la tasa de interés, mediante ajustes por cantidad.

Estas imperfecciones de los mercados financieros tienen claras repercusiones en la movilidad internacional del capital, ya que dificultan la materialización de los beneficios potenciales de los flujos de recursos. Primero, en lo que se refiere al papel del ahorro externo, tal vez no siempre se den las condiciones ideales. Los países pueden experimentar una sustitución de ahorro interno por ahorro externo, y las inversiones pueden no ser siempre eficientes, o no estar suficientemente concentradas en bienes transables para generar los flujos de divisas necesarios (Devlin, FfrenchDavis y Griffith-Jones, 1994). Como se sabe, las entradas de capitales tienden a reducir las tasas de interés y a estimular el gasto interno. La parte que va a bienes transables tiende a acomodarse a través de un mayor déficit corriente. Sin embargo, también se produce un exceso de demanda de bienes no transables con tendencia a una apreciación real, un mayor sector no transable, un sector transable pequeño y un gran déficit comercial.

Segundo, con respecto a la diversificación asociada a la libre movilidad de activos financieros internacionales como las inversiones de cartera, cabe señalar que éstas se caracterizan por sus bruscas reversiones. 
En contraste con otras formas de capital - como la inversión extranjera directa, los préstamos concedidos por instituciones financieras transnacionales o los préstamos bancarios de largo plazo-, las inversiones de cartera tienen incorporado el riesgo de fuertes reversiones en el corto plazo. Esta volatilidad puede provocar grandes fluctuaciones en los tipos de cambio, en las tasas de interés, o en ambos. Lo imprevisible de sus efectos sobre los precios puede desalentar a los inversionistas internos y externos de ampliar sus inversiones en el país, y si las fluctuaciones en las tasas de interés o tipos de cambio son muy acentuadas, pueden afectar a la economía en su conjunto (Devlin, FfrenchDavis y Griffith-Jones, 1994).

Asimismo, estos elementos pueden servir para impedir la entrada de capitales compensatorios en cantidades que obliguen a severos ajustes económicos debidos a discrepancias entre el ingreso y el gasto, llevando a las economías en desarrollo a incurrir en considerables costos cuando se enfrentan a condiciones externas adversas. Para tales economías, cuyas reservas internacionales se agotan con facilidad, cualquier conmoción externa negativa que reduzca la afluencia de capitales forzará una inmediata caída del gasto interno, en un esfuerzo por recuperar el equilibrio en la balanza de pagos. La producción casi con seguridad disminuirá, dependiendo de los elementos de irreversibilidad y dadas las naturales rigideces que dificultan la reasignación de recursos, iniciándose así una tendencia al descenso de la inversión.

Los factores descritos se han manifestado en toda su magnitud durante la reciente crisis asociada al "efecto tequila" que ha afectado con singular fuerza a México y Argentina.

\section{El papel del sistema financiero en la intermediación de movimientos de capitales}

En lo que toca al ámbito microeconómico, la afluencia de capital al país acrecentará el volumen đe fondos intermediados a través del sistema financiero $y$, por ende, el volumen de activos y pasivos internos.

La experiencia muestra que en un sistema financiero que ha sido recientemente liberalizado para operar en condiciones de mercado, en el cual la supervision se encuentra en vías de adecuarse al nuevo escenario, y que además recibe fuertes entradas de capitales, las intervenciones en el mercado cambiario que no sean plenamente esterilizadas exponen a la banca a un mayor riesgo crediticio como consecuencia de la expansión de sus activos. Se ha observado que el rápido incremento de las colocaciones de los bancos suele ir unido al relajamiento de los estándares de evaluación del riesgo crediticio. Este aumento suele concentrarse en unos pocos sectores y cualquier reversión del ciclo suele ir acompañada de un deterioro en el riesgo de la cartera de inversiones de las instituciones financieras (Gavin y Hausmann,1995).

Por otra parte, en las economías que se vieron más afectadas por el contagio de la crisis mexicana, la baja calidad de los activos de los bancos limitó la aplicación de políticas de ajuste basadas en incrementos de las tasas de interés como respuesta a la pérdida de confianza en la moneda local. Así, un efecto indirecto de un alza brusca de las tasas de interés de corto plazo fue el aumento de los activos no rentables de los bancos. Dado que los créditos de los bancos locales son una fuente fundamental de financiamiento en las economías emergentes, cualquier aumento sostenido de las tasas de interés tendrá seguramente un efecto fuertemente contractivo, deteriorará la calidad media de los deudores y alentará un fenómeno de selección adversa, mermando la solvencia de las instituciones. En estas circunstancias, el Banco Central tendrá un menor espacio de maniobra para recurrir a alzas de esas tasas (FM1, 1995).

\section{Movimientos de capitales Internacionales en los años noventa}

Según antecedentes preliminares elaborados por organismos internacionales, durante 1995 la transferencia neta de recursos financieros hacia América Latina, por primera vez desde 1990 , fue negativa: -10600 millones de dólares (CEPAL, 1995; FMI, 1995). Es interesante anotar, sin embargo, que la tendencia ascendente de la entrada de capitales internacionales comenz 6 a revertirse en 1994, lo que significa que otros factores se suman a la crisis mexicana para explicar el comportamiento de esas corrientes de recursos. Cabe señalar que la transferencia positiva a la región fue de 11500 millones de dólares en 1994 y de 34000 millones en 1993. Estas cifras excluyen el financiamiento excepcional de la balanza de pagos, que en Argentina, México y Nicaragua en conjunto alcanzó a 29100 millones.

Algunos de los factores que contribuyeron a explicar el fuerte aumento de la afluencia de capitales hacia países emergentes entre 1991 y 1993 permiten entender el cambio en el comportamiento de los movimientos de capital en 1994 y 1995 (Corbo y Hernández,1996). En 1994 la aceleración del crecimiento y el alza de las tasas de interés en Estados 
Unidos, junto con un ajuste en las condiciones financieras de varias importantes economías industrializadas, llevaron a los inversionistas a readecuar su cartera global en desmedro de las economías emergentes (Griffith-Jones,1994). Si a los factores anteriores se agrega que los efectos de la crisis mexicana minaron la confianza de los inversionistas en la fortaleza de las economías regionales y en la solidez de sus equilibrios macroeconómicos fundamentales, es fácil comprender la transferencia negativa de fondos hacia América Latina en 1995.

Sin perjuicio de lo anterior, una serie de transformaciones ha configurado un nuevo escenario institucional, influyendo decisivamente en la forma en que los capitales han respondido en los años noventa a las condiciones financieras de los países desarrollados o a desequilibrios macroeconómicos básicos de las economías emergentes. En primer lugar, la liberalización de los movimientos de capitales en las mayores de estas economías ha significado una creciente integración de sus mercados en los mercados financieros globales. Segundo, el peso adquirido por los títulos financieros en las finanzas internacionales ha hecho que los créditos bancarios sindicados estén dando paso a los bonos y acciones como instrumentos preferidos para la transferencia de capital hacia los mercados emergentes (Hale,1995). Y tercero, el crecimiento de los inversionistas institucionales ha significado que las corrientes de capital hacia economías emergentes están ahora determinadas por consideraciones de liquidez y rentabilidad, más que por relaciones bancarias estructuradas a largo plazo (FMI,1995).

Estos cambios en el entorno han tenido al menos dos consecuencias importantes. Por un lado, la liberalización de las transacciones transfronterizas y la progresiva integración a los mercados de capitales globales, han permitido que las empresas e individuos de las mayores economías emergentes accedan a transacciones de bajo costo en los mercados bancarios y de capitales del ámbito internacional, no sólo a nivel mayorista sino también al detalle. Si los residentes dudan de la sustentabilidad del régimen de política cambiaria vigente en el país, pueden ajustar la denominación de monedas de sus activos financieros de manera rápida y a bajo costo (FMI, 1995).

Por otro lado, la presente incorporación de las economías emergentes en los mercados de capitales globales los ha hecho más vulnerables a acontecimientos externos, como los cambios en el ciclo de las economías industrializadas y los problemas en cualquiera de los mayores mercados financieros. Aunque experiencias recientes demuestran que los problemas de cualquier economía importante se extienden a nivel regional y global, cabe destacar que una vez controlado el pánico inicial, los mercados discriminan, aunque imperfectamente, según la solidez y estabilidad de las variables fundamentales de las economías.

En términos generales, países con bajas tasas de ahorro, grandes déficit en cuenta corriente, sistemas bancarios débiles y cuantiosa deuda de corto plazo experimentaron mayores presiones externas que los países con variables fundamentales sólidas y estables. En definitiva, el espacio para aplicar políticas económicas desalineadas de esas variables se ha estrechado notoriamente, por lo que el desafío actual es el de mantener equilibradas las economías antes que los inversionistas obliguen a soluciones más costosas. Esto apunta a la necesidad de contar con políticas que regulen los movimientos de capitales y así permitan manejar con eficiencia los riesgos macroeconómicos y financieros asociados a ellos, sin privarse de sus beneficios.

\section{III}

\section{La política de regulación de los movimientos de capitales internacionales en la economía chilena}

La renovada afluencia de capitales hacia las economías emergentes ha tenido favorables repercusiones en los niveles de actividad e inversión de éstas y, en contra- partida, ha causado presiones que han afectado la oferta monetaria y la tendencia hacia la apreciación cambiaria. 
CUADRO 2

Chlle: Movimlentos netos de capitales

(Como porcentaje del PIB)

\begin{tabular}{lrrrrrr}
\hline & 1991 & 1992 & 1993 & 1994 & 1995 & $1990-1995$ \\
\hline Movimiento neto de capitales & 2.3 & 6.7 & 6.0 & 8.9 & 1.7 & 6.0 \\
Inversión extranjera directa & 1.2 & 1.5 & 2.5 & 3.4 & 1.5 & 2.3 \\
Mediano y largo plazo & -0.2 & 0.7 & 1.1 & 2.6 & -0.3 & 0.9 \\
Corto plazo & 1.3 & 4.5 & 2.4 & 2.9 & 0.5 & 2.8 \\
\hline
\end{tabular}

Fuente: Banco Central de Chile.

El sistema bancario, por su parte, ha estado en el centro de la crisis asociada al "efecto tequila", en la medida en que ha tenido que absorber los efectos de las alzas de tasas de interés requeridas para defender el valor de la moneda del país. La liberalización financiera combinada con entradas de capital carentes de adecuada supervision ha mostrado ser terreno fértil para futuras crisis bancarias (Rojas-Suárez y Weisbrod, 1995).

En el caso chileno, las medidas para promover y diversificar las exportaciones están en el centro de la estrategia de desarrollo. Una variable clave al respecto ha sido un tipo de cambio estable y compatible con el equilibrio externo de mediano plazo. Asimismo, las continuas reducciones de la inflación han desempeñado un papel esencial en el proceso de desartollo chileno, ya que es reconocido que las menores tasas de inflación favorecen la eficiencia en la asignación de recursos y estimulan la competitividad de la economía.

En 1990-1995 la afluencia neta de capitales a Chile fue de $6 \%$ de su PIB, como promedio anual (cuadro 2). Las corrientes de inversión extranjera directa y de mediano y largo plazo no sólo representaron más del $50 \%$ de las entradas netas de capitales en el período, sino que también marcaron una tendencia ascendente en el tiempo. Es importante señalar que la inversión extranjera directa se incrementó gradualmente cada año, alcanzando a un 3.4\% del PIB en 1994.

En Chile esto condujo a un dilema de política: ¿cómo conciliar las reducciones de la inflación con la mantención de un tipo de cambio real compatible con un déficit de la cuenta corriente de la balanza de pagos sustentable en el mediano plazo?

En 1995, la economía chilena también vio disminuir la entrada neta de capitales, la que alcanzo al $1.7 \%$ del pIB. De esto, la inversión extranjera directa alcanzó al $1.5 \%$ del PIB. Aunque esta inversión mostró un aumento del $19 \%$ sobre el año anterior, se vio contrarrestada por la caída de los capitales de cartera en los tres primeros trimestres del año. Por su parte, los préstamos de mediano y largo plazo llegaron a un $-0.3 \%$, fundamentalmente debido a prepagos de la deuda externa a organismos multilaterales por un total de 1391 millones de dólares, efectuados por el fisco y el Banco Central.

Lo interesante del caso de Chile es que, no obstante lo anterior, su economía no se vio mayormente afectada en sus resultados macroeconómicos. Asimismo, las perspectivas para 1996 apuntan a una recuperación neta de la entrada de capitales, tanto en lo que se refiere a inversión extranjera directa como a capitales de cartera.

Los objetivos estratégicos de la política de regulación de los movimientos de capitales aplicada en la economía chilena son, por un lado, evitar los trastornos que pueden causar las entradas de capitales en el gasto, la inflación y el tipo de cambio, y, por ende, que estas variables se vean desequilibradas o desviadas de su tendencia de mediano plazo; y por otro - lo que ha sido un objetivo central del manejo económico-, evitar a través de adecuadas normativas prudenciales y de un activo seguimiento, que la creciente intermediación de capitales por parte de la banca pueda afectar la solvencia y estabilidad del sistema financiero. Todo esto apunta a garantizar una eficiente cobertura del riesgo crediticio y financiero de las operaciones bancarias.

\section{Aspectos macroeconómicos}

Como se señal6, el objetivo central de la política macroeconómica chilena ha sido evitar las distorsiones que pueden ocasionar las entradas de capitales en la inflación y el tipo de cambio.

El instrumento esencial de la política antiinflacionaria chilena es el manejo de la tasa de interés de corto plazo ofrecida por los títulos del Banco Central. Este instrumento, unido a la política fiscal, contribuye a 
CUADRO 3

Chile: Cuentas externas y flujos de capitales

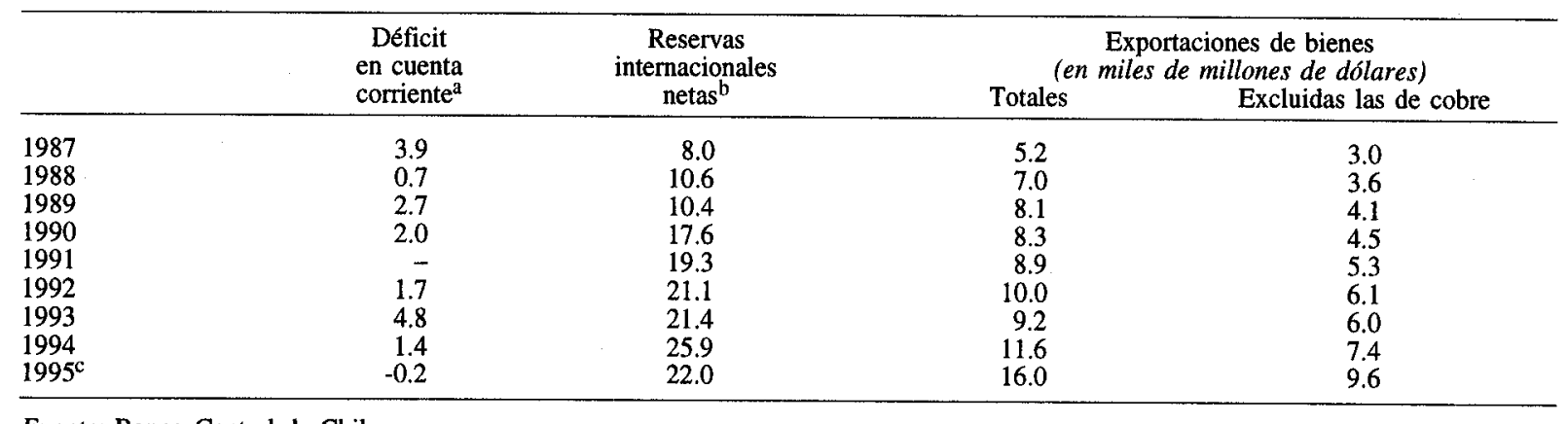

Fuente: Banco Central de Chile.

a Como porcentaje del PIB.

b Como porcentaje de exportaciones y servicios distintos de los de factores.

c Cifras preliminares.

mantener el gasto agregado y la producción alineados con un crecimiento real del producto que sea sostenible en el largo plazo (dadas las tasas de crecimiento de la fuerza de trabajo, la inversión y la productividad).

Este enfoque ha permitido a la economía chilena bajar su tasa de inflación en forma gradual pero sostenida y alcanzar en 1995, por segundo año consecutivo, tasas de un solo dígito.

La creciente apertura de la cuenta de capitales y la consecuente integración del mercado financiero del país en los mercados globales, han disminuido el margen de autonomía de la política sobre tasas de interés, reduciendo la diferencia que puede existir entre la tasa de interés interna y la externa. ${ }^{2}$

En este contexto, la opción de mantener una política de reducción sistemática de la inflación a través de la tasa de interés obliga a utilizar un conjunto de herramientas para lograr una baja progresiva de la inflación sin afectar el tipo de cambio de equilibrio de mediano plazo. Con este propósito en Chile se ha aplicado una combinación de políticas que abarca cuatro elementos centrales: intervención con esterilización, revaluaciones reales graduales, desincentivos a la entrada de capitales de corto plazo y flexibilización de las salidas de capitales (Ffrench-Davis, Agosin y Uthoff, 1995).

\footnotetext{
${ }^{2}$ En países con tipo de cambio flexible, la apreciación del tipo de cambio nominal en períodos de fuerte entrada de capitales puede aislar los efectos monetarios y crediticios de tales corrientes. Sin embargo, los movimientos abruptos en el tipo de cambio real pueden imponer fuertes ajustes a la economía, particularmente si la apreciación se revierte cuando el capital se retira.
}

La intervención del Banco Central se ha reflejado en un fuerte incremento de las reservas internacionales (de 3000 millones de dólares en diciembre de 1989 a 14800 millones a fines de 1995). Los aumentos en la oferta monetaria asociados a la compra de divisas en el mercado pueden conducir a niveles de liquidez superiores a los requeridos para cumplir con las metas oficiales de actividad e inflación (cuadro 3).

Para contrarrestar los efectos monetarios de la acumulación de reservas el Banco Central de Chile ha recurrido a la esterilización, a través de las operaciones de mercado abierto. Sin embargo, son conocidos los límites que ésta puede tener para eliminar los efectos de la intervención. En la medida en que la colocación de instrumentos de deuda del Banco Central conduce a fortalecer la diferencia entre las tasas de interés internas y las externas, una esterilización plena y prolongada puede distorsionar las primeras lo suficiente como para atraer más capitales y, peor aún, elevar la participación de los recursos de corto plazo. La esterilización tiene también costos cuasi fiscales que resultan de la diferencia entre las tasas de interés obtenidas por las reservas internacionales y la tasa pagada por el Banco Central para la colocación interna de sus instrumentos financieros.

El proceso ha ido acompañado por graduales revaluaciones reales del peso, las que han permitido aliviar las presiones cuasi fiscales derivadas de la entrada de capitales. En enero de 1992, el llamado "dólar acuerdo" fue revaluado en 5\%, y la banda de flotación del dólar en el mercado cambiario formal fue ampliada hasta $\pm 10 \%$. Abruptamente, el tipo de cambio observado cayó un $9 \%$, al filo del $10 \%$, derivado de la revaluación y de la flexibilización de la banda. 
Más tarde, a fines de 1994, el dólar acuerdo fue revaluado en un $10 \%$ adicional.

Desde mediados de 1991 Chile ha aplicado asimismo controles no cuantitativos de capital (a la Tobin, 1978), lo que desincentiva la afluencia de capitales de corto plazo. El principal mecanismo utilizado ha sido un encaje del $30 \%$ sobre el endeudamiento externo de bancos y empresas, que tiene vigencia únicamente por un año (cuadro 4). Este encaje permite anpliar el margen de maniobra de la política monetaria para el control del gasto mediante las tasas de interés, y reducir el impacto negativo de la esterilización sobre la entrada de capitales. Es interesante señalar que la composición de las entradas netas de recursos en la economía chilena ha cambiado en los años noventa: la participación de los capitales de largo plazo ha aumentado, lo que en parte se explica por dichos controles a la entrada de capitales.

Por otra parte, un rasgo distintivo de la política chilena de regulación de los movimientos de capitales desde 1991 ha sido el de flexibilizar las salidas de capitales y facilitar la repatriación temprana de ellos a fin de aliviar las presiones monetarias y cambiarias asociadas a la entrada de divisas. Con este propósito, se ha autorizado a los fondos de pensiones a invertir en el exterior, con un límite que se ha ido ampliando progresivamente hasta llegar al $9 \%$ de cada fondo en 1996, junto con flexibilizarse los tipos de instrumentos permitidos para dichas inversiones.

También se han flexibilizado las regulaciones sobre las inversiones directas de agentes del país en el exterior. Anteriormente, el capítulo XII de la normativa del Banco Central estipulaba que la compra de divisas destinadas a inversiones en el exterior debía efectuarse a través del mercado cambiario formal -donde los principales agentes participantes son los ban$\cos -$, con la autorización del Banco Central. En abril

CUADRO 4

Chlle: Costo del crédlto externo sujeto a encale (Promedios anuales)

\begin{tabular}{lccc}
\hline & LIBOR & Hasta 1 año & Hasta 3 años \\
\hline 1991 & 6.1 & 7.9 & 6.8 \\
1992 & 3.9 & 5.8 & 4.7 \\
1993 & 3.4 & 5.1 & 4.1 \\
1994 & 5.1 & 7.5 & 6.0 \\
1995 & 6.1 & 9.1 & 7.3 \\
\hline
\end{tabular}

Fuente: Banco Central de Chile.

a También incluye el impuesto sobre los intereses.
CUADRO 5

Chile: Ahorro interno y superávit fiscal (Como porcentaje del PIB)

\begin{tabular}{lccc}
\hline & $\begin{array}{c}\text { Ahorro } \\
\text { interno }\end{array}$ & $\begin{array}{c}\text { Ahorro } \\
\text { públiç }\end{array}$ & $\begin{array}{c}\text { Superávit } \\
\text { fiscal }\end{array}$ \\
\hline 1990 & 24.2 & 2.5 & 0.8 \\
$199 \mathrm{I}$ & 24.1 & 3.7 & 1.5 \\
1992 & 24.8 & 4.9 & 2.2 \\
1993 & 23.9 & 4.8 & 1.9 \\
1994 & 25.4 & 4.8 & 1.7 \\
$1995^{\mathrm{a}}$ & 27.6 & 5.5 & 2.6 \\
\hline
\end{tabular}

Fuente: Banco Central de Chile.

a Cifras preliminares.

de 1991, esta cláusula fue modificada para permitir la compra de divisas en el mercado informal, sin más exigencia que la de informar al Banco Central.

En cuanto al sistema financiero, existe un proyecto de modificación de la ley de bancos que permitirá una amplia internacionalización de la banca chilena, tanto a través de colocaciones transfronterizas como de inversiones directas de los bancos en el exterior, vía sucursales o subsidiarias (Larraín, 1995). Asimismo, recientemente fueron promulgadas las leyes que permitirán la existencia de fondos mutuos especializados en fondos de inversión en el exterior. Dichas regulaciones apuntan a estimular la salida de capitales y a diversificar más el riesgo de la economía chilena.

Este proceso se ha basado en una alta tasa de ahorro interno, apoyada en una política de austeridad fiscal, y en los recursos derivados del sistema privado de pensiones (Uthoff y Titelman,1996). Se ha contribuido así a disminuir las presiones al alza de tasas de interés derivadas de la esterilización, al desincentivarse la entrada de capitales de corto plazo y disminuir la vulnerabilidad de la economía ante las reversiones de movimientos de capitales externos. Según estimaciones preliminares, hacia 1995 la tasa de ahorro como porcentaje del PIB sería de $27.4 \%$, del cual un $27.6 \%$ correspondería a ahorro interno (cuadro 5). En 19901995 el ahorro externo fue, en promedio, del orden del $2 \%$ del PIB. Por su parte, la política fiscal ha conducido sistemáticamente a cuentas superavitarias. En los años indicados hubo en promedio un ahorro fiscal anual de $4.4 \%$ del PrB, y un superávit global del $1.8 \%$ del PIB.

\section{Aspectos financieros}

A diferencia de lo sucedido en otros países de la región, el sistema bancario chileno no se vio afectado por la reversión de los flujos de capitales. Si bien es cier- 
to que esto se debió en gran medida a que no sufrieron ni la estabilidad macroeconómica ni el crecimiento, también lo es que la "profundidad" y solvencia del sistema financiero han contribuido a un proceso sano de intermediación de los flujos de capitales y a la mantención de estándares crediticios adecuados para que la expansión de las colocaciones guarde relación con el crecimiento de las variables fundamentales de la economía.

La experiencia en la evaluación de riesgos crediticios y financieros en una economía de mercado, que ha acumulado por largos años el sistema financiero chileno, y la presencia de un organismo de supervisión bancaria fuerte y altamente calificado -una de las lecciones importantes de la crisis de la deuda en 1982 - ha permitido a la banca intermediar la creciente afluencia de recursos asociada a los capitales externos, sin que ello conduzca a un deterioro de la calidad de los activos ni a incrementos insostenibles de las colocaciones.

En los esquemas de supervisión bancaria debe hacerse hincapié en su carácter prudencial, es decir, en su capacidad de prever los problemas y tomar medidas correctoras antes de que ellos se materialicen. $\mathrm{La}$ legislación bancaria chilena contiene elementos que contribuyen a esto: la facultad de supervisar los activos y los requerimientos de reservas; los límites y las drásticas sanciones establecidos para operaciones con

\section{IV}

\section{Conclusiones}

Las imperfecciones que caracterizan la operación de los mercados financieros internacionales, las transformaciones en la naturaleza de los movimientos de capitales y la creciente integración de las economias emergentes en los mercados mundiales, ponen de relieve la importancia de una política de regulación de flujos de capitales que pueda cubrir los riesgos macroeconómicos y financieros vinculados a las entradas de recursos externos, sin que se pierdan los beneficios asociados a ellos.

En el nuevo marco institucional que está condicionando los movimientos de capitales internacionales, la vulnerabilidad de las economías emergentes frente a reversiones en las entradas de capitales se ha acentuado considerablemente, reduciendo el margen para partes relacionadas; los mecanismos automáticos de ajuste patrimonial cuando el capital económico cae bajo los mínimos exigidos por el regulador, y las facultades para congelar las operaciones de las instituciones bancarias, impedir transferencias de fondos fuera del banco y restringir el pago de dividendos a las instituciones que no cumplan con los niveles de capitalización adecuados (Larraín, 1994).

Para que el mercado pueda colaborar en la fiscalización de las instituciones financieras, es fundamental contar con un esquema de seguros que proteja la estabilidad del sistema de pagos y reduzca la exposición de las instituciones a las corridas, pero que a la vez tenga un carácter acotado, a fin de estimular a los depositantes a evaluar por sí mismos la condición de los bancos. En Chile existe una garantía total para los depósitos a la vista, avalada por el Banco Central, el que como contrapartida exige que en el activo se mantengan instrumentos libres de riesgo (reserva técnica); además, existe una garantía estatal acotada hasta el 90\% del resto de los depósitos por un monto de hasta 3000 dólares aproximadamente.

La transparencia de la información también es fundamental para evitar cualquier tipo de "maquillaje contable" y para que el propio mercado pueda vigilar la situación de los bancos. En el caso chileno, la transparencia es un bien jurídico y existe pleno acceso del regulador a toda la información de las instituciones. errores en el manejo de las políticas económicas y para desequilibrios en los fundamentos macroeconómicos y microeconómicos de las economías emergentes.

Los objetivos estratégicos de la política de regulación de los movimientos de capitales aplicada en Chile son, por un lado, minimizar las distorsiones que pueden generar esos movimientos en la tasa de interés, la inflación y el tipo de cambio y, por otro, impedir que la intermediación de capitales a través de la banca conduzca a una expansión desenfrenada del crédito que pueda afectar la estabilidad y la solvencia del sistema financiero.

Entre los instrumentos de esta política están la intervención en el mercado de divisas, unida a la esterilización parcial de los efectos monetarios de dicha 
intervención, y moderadas revaluaciones reales del peso que permiten aliviar los costos asumidos por el Banco Central en su proceso de esterilización.

Otro componente de la regulación de los movimientos de capitales ha sido la aplicación de controles no cuantitativos destinados a desalentar la entrada de capitales de corto plazo. El objetivo de esta medida no es distorsionar los valores tendenciales de la economía, sino más bien ampliar el margen de maniobra de la política monetaria para mantener el gasto alineado con el producto potencial.

La misma importancia que los componentes anteriores tiene la política de flexibilización de las salidas de capital, fundamentalmente a través de las inversiones directas de agentes internos en el exterior y de la regulación de las operaciones de los inversionistas institucionales. Destacan en esta política el actual proyecto de ley de bancos, que permitirá ampliar considerablemente las operaciones externas del sistema financiero, y la creación de fondos mutuos especializados en invertir en el exterior.
El trasfondo de esta regulación de los movimientos de capitales ha sido la consecución de altas tasas de ahorro interno, que ha contribuido a disminuir la vulnerabilidad de la economía chilena a los movimientos de capitales externos. En esta línea, la aplicacion de una política fiscal austera también ha sido crucial para acomodar los efectos monetarios de las entradas de capitales y ha contribuido a que la tasa de ahorro interno sea alta.

Por último, un aspecto central de política ha sido el de mantener adecuadas exigencias de carácter prudencial que garanticen la capacidad de los bancos de administrar los distintos tipos de riesgos. Si el sistema bancario tiene una adecuada capacidad de gestión de riesgos, junto con la debida "profundidad" y flexibilidad, y si las autoriđades de supervisión están bien equipadas y calificadas, un sistema financiero internacionalmente activo no se verá adversamente afectado por el fortalecimiento de su rol de intermediación.

\section{Bibliografia}

CEPAL (Comisión Económica para América Latina y el Caribe) (1995): Balance preliminar de la economía de América Latina y el Caribe 1995. LC/G.1892-P, Santiago de Chile, diciembre. Publicación de las Naciones Unidas $\mathrm{N}^{\circ}$ de venta S.95.II.G.18.

Corbo, V. y L. Hernández (1996): Macroeconomic adjustment to capital inflows: Lessons from recent Latin American and East Asian experience. The World Bank Research Observer. vol.11, $\mathrm{N}^{\circ}$ 1. Washington, D.C., Banco Mundial, febrero.

Devlin, R., R. Ffrench-Davis y S. Griffith-Jones, (1994): Repunte de los fujos de capital y el desarrollo: implicancias para las polfticas económicas. Serie Financiamiento del desarrollo, $N^{\circ} 26$, LC/L.859, Santiago de Chile, CEPAL, agosto.

Ffrench-Davis, R., M. Agosin y A. Uthoff, (1995): Movimiento de capitales, estrategia exportadora y estabilidad macroeconómica en Chile, R.Ffrench-Davis y S. Griffith-Jones (comps.): Las nuevas corrientes financieras hacia América Latina: fuentes, efectos y políticas, México, D.F., Fondo de Cultura Económica (FCE)

FMI (Fondo Monetario Internacional) (1995); International Capital Markets: Developments, Prospects and Policy issues. Washington, D.C., agosto.

Gavin, M. y R. Hausmann (1995): The roots of banking crises: The macroeconomic context, preparado para la Conferencia del Grupo de los Treinta sobre las crisis bancarias en América Latina, Washington, D.C., Banco Interamericano de Desarrollo (BID), septiembre, mimeo.
Griffith-Jones, S. (1994): Corrientes de fondos privados europeos hacia America Latina: Hechos y planteamientos. Serie Financiamiento del desarrollo, $\mathrm{N}^{\circ} 24, \mathrm{LC} / \mathrm{L} .855$, Santiago de Chile, CEPAL.

Hale, D. (1995); Lessons from the Mexican peso crisis of 1995 for international economic policy, Viena, Institute for International Economics (IIE)/Oesterreichische Nationalbank, septiembre, mimeo.

Larraín, C. (1994): Modernización de la supervisión bancaria, Revista de la CEPAL, No 54, LC/G.1845-P, Santiago de Chile, CEPAL.

(1995): Internacionalización y supervisión de la banca en Chile, Estudios Públicos, No 60, Santiago de Chile, Centro de Estudios Públicos (CEP).

Rojas-Suárez, L. y S. R. Weisbrod (1995): Financial Fragilities in Latin America. The 1980s and 1990s. Occasional Paper, $N^{\circ} 132$, Washington D.C., FMI.

Stiglitz, J. E (1993): The role of the State in financial markets. Proceedings of the World Bank Annual Conference on Development Economics, vol, 2, Washington, D.C., Banco Mundial.

Tobin, J. (1978): A proposal for international monetary reform, Eastern Economic Journal, $\mathrm{N}^{\circ} 4$.

Uthoff, A. y D.Titelman (1996): The relation between foreign and national savings under financial liberalization, documento preparado para el taller OCDE/CEPAL Capital Flows and Investment Performance in Latin America, Santiago de Chile, CEPAL, marzo, mimeo. 